\title{
ARTICLE
}

Clinical Study

\section{Routine blood investigations have limited utility in surveillance of aggressive lymphoma in asymptomatic patients in complete remission}

\author{
Eliza A Hawkes ${ }^{1,2,3}$, Zoe Loh ${ }^{1,4}$, Ortis Estacio ${ }^{4}$, Geoff Chong ${ }^{1,2}$, Francis J Ha ${ }^{5}$, Michael Gilbertson ${ }^{6}$ and Andrew Grigg ${ }^{1,2,4}$
}

BACKGROUND: Patients with aggressive lymphoma achieving complete remission (CR) after first-line combination chemotherapy undergo regular surveillance to detect relapse. Current international guidelines recommend routine follow-up blood tests in this context, but evidence supporting this practice is limited.

METHODS: We conducted a multi-centre retrospective analysis of all patients diagnosed with aggressive lymphoma treated with curative-intent chemotherapy who achieved CR for at least 3 months between 2000 and 2015. An abnormal blood test was defined as any new and unexplained abnormality for full blood examination, lactate dehydrogenase or erythrocyte sedimentation rate. RESULTS: Three hundred and forty-six patients attended a total of 3084 outpatient visits; blood tests were performed at $90 \%$ of these appointments. Fifty-six (16\%) patients relapsed. Routine laboratory testing detected relapse in only three patients $(5 \%$ of relapses); in the remaining patients, relapse was suspected clinically $(80 \%)$ or detected by imaging (15\%). The sensitivity of all blood tests was $42 \%$ and the positive predictive value was $9 \%$. No significant difference in survival was shown in patients who underwent a routine blood test within 3 months prior to relapse versus those who did not ( $p=0.88$ ).

CONCLUSIONS: Routine blood tests demonstrate unacceptably poor performance characteristics, have no impact on survival and thus have limited value in the detection of relapse in routine surveillance.

British Journal of Cancer (2018) 119:546-550; https://doi.org/10.1038/s41416-018-0183-x

\section{INTRODUCTION}

While the majority of patients with aggressive lymphomas achieve complete remission (CR) with anthracycline-based combination chemotherapy, up to $50 \%$ of patients will relapse. ${ }^{1-3}$ As a significant proportion of patients who relapse are considered for salvage chemotherapy and curative-intent autologous stem cell transplant, surveillance after first-line therapy is recommended. ${ }^{4}$

In patients achieving $C R$, the optimal frequency, duration and type of surveillance are not established. As follow-up imaging is associated with increased radiation-related risk and minimal benefit in asymptomatic patients, such surveillance is no longer routine. $^{5-7}$ Regular laboratory testing (Labs) still features in internationally recognised surveillance guidelines, despite limited evidence for their use in detecting relapse. ${ }^{7-10}$ Studies conducted prior to modern treatment response assessments and routine rituximab administration suggested that lactate dehydrogenase $(\mathrm{LDH})$ and erythrocyte sedimentation rate (ESR) may be useful as surveillance tools, and that, more recently, the absolute lymphocyte count (ALC) and lymphocyte-monocyte ratio (LMR) have shown promise in small series. ${ }^{1-13}$ However, large-scale data are lacking, particularly in the era of positron emission tomography (PET)-defined complete metabolic response (CMR).
Clinically significant scan-related anxiety has been established in both lymphoma and solid malignancies ${ }^{14,15}$; this is reported in up to $80 \%$ of patients and does not abate over time. It is likely that blood tests have similar consequences. In addition, routine laboratory investigations have cost implications and are potentially falsely reassuring if normal. Abnormal results are also associated with the potential for expensive, unnecessary additional investigations.

To evaluate the role of routine blood testing in follow-up of patients with aggressive lymphoma, we analysed the use of blood tests in patients with high-grade lymphomas undergoing surveillance after achieving CMR from curative-intent combination chemotherapy at three large Australian cancer centres. In particular, we examined the utility of routine tests for the detection of relapse in the absence of clinical symptoms or signs, and whether performing such tests was associated with significant differences in post-relapse survival.

\section{METHODS}

Patients

Patients were identified from an electronic database at three institutions. Eligible patients were aged 16 years or older, with a

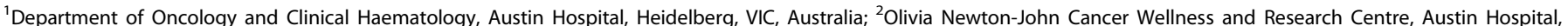

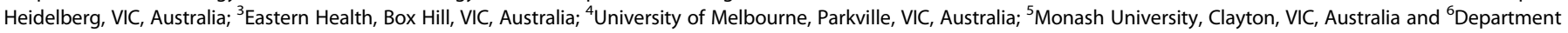
of Haematology, Monash Medical Center, Monash Health, Clayton, VIC, Australia

Correspondence: Eliza A Hawkes (eliza.hawkes@onjcri.org.au)

These authors contributed equally: Eliza A. Hawkes, Zoe Loh

Received: 16 February 2018 Revised: 3 May 2018 Accepted: 25 June 2018

Published online: 23 July 2018 
Table 1. Baseline demographics

\begin{tabular}{|c|c|}
\hline & All patients $n=346(\%)$ \\
\hline Median age (range) & $54(17-91)$ \\
\hline \multicolumn{2}{|l|}{ Gender } \\
\hline Male & $202(58)$ \\
\hline Female & $144(42)$ \\
\hline \multicolumn{2}{|l|}{ Stage } \\
\hline I-II & $168(49)$ \\
\hline II-IV & $178(51)$ \\
\hline \multicolumn{2}{|l|}{ Subtype } \\
\hline DLBCL & $187(54)$ \\
\hline $\mathrm{HL}$ & $119(34)$ \\
\hline $\mathrm{BL}$ & $22(6)$ \\
\hline $\mathrm{TCL}$ & $18(5)$ \\
\hline B symptoms & 201(58) \\
\hline$>1$ extranodal site & $68(20)$ \\
\hline Bone marrow involvement & $39(11)$ \\
\hline \multicolumn{2}{|l|}{ Performance status } \\
\hline $0-1$ & $306(88)$ \\
\hline$\geq 2$ & $40(12)$ \\
\hline \multicolumn{2}{|l|}{ Prognostic score } \\
\hline Low risk ${ }^{a}$ & $280(81)$ \\
\hline High risk ${ }^{b}$ & $66(19)$ \\
\hline \multicolumn{2}{|l|}{ Treatment } \\
\hline Chemotherapy alone & $238(69)$ \\
\hline Chemotherapy and radiotherapy & $108(31)$ \\
\hline
\end{tabular}

a Low risk $=$ International Prognostic Index for DLBCL and TCL: 0-2; Hasenclever score for HL: 0-3; prognostic score for Burkitt's lymphoma: $0-2$. ' ${ }^{b}$ High risk = International Prognostic Index for DLBCL and TCL: >3; Hasenclever score for $\mathrm{HL}$ : $>4$; prognostic score for Burkitt's lymphoma: >3

documented histological diagnosis of diffuse large $B$ cell lymphoma (DLBCL), Hodgkin's lymphoma $(\mathrm{HL})$, T cell lymphoma (TCL) or Burkitt lymphoma (BL) who received curative-intent firstline treatment and in documented $C R$ on PET/CT for at least 3 months after completion of therapy. Those with primary progressive lymphoma, in partial remission (PR) at the end of first-line treatment, primary central nervous system lymphoma, HIV-associated lymphoma and transformation from indolent subtypes were excluded from the analysis.

All information was obtained from electronic patient records. Data were collected on gender, age, disease stage, comorbidities, presence of B symptoms, Eastern Cooperative Oncology Group performance status, extranodal sites of disease, prognostic score and first-line chemotherapy treatment. Details of each outpatient appointment were recorded, including pathology results, presence of relevant symptoms and/or clinical signs (the absence of both was deemed 'asymptomatic'), whether the visit was scheduled or unplanned, and outcomes including routine subsequent visit, earlier planned review and results of additional investigations ordered. Relapse date, site and method of diagnosis, any further treatment and date of death or last follow-up were also documented.

Patient follow-up at all three institutions was according to institutional guidelines as follows: 3-monthly for the first 2 years after completion of therapy, and then every 6 to 12 months for the following 3 years for at least 5 years in total. Blood tests were recommended but performed at the treating physician's discretion. Imaging was also performed according to the treating physician's discretion but removed from the institutional guidelines in 2014.

Statistical analysis

The primary endpoints of the study were to assess whether full blood examination (FBE: haemoglobin, white cell count and platelet count), LDH, ESR, ALC, absolute monocyte count (AMC) and LMR during follow-up are reliable markers to predict relapse. Secondary endpoints include methods of relapse detection, eventfree survival (EFS) and overall survival (OS). EFS was defined as the period from the date of diagnosis until relapse, disease progression or death from any cause. OS was measured from the date of diagnosis until death from any cause.

Laboratory results were considered abnormal if all of the following were fulfilled: (a) any component of FBE, LDH or ESR fell outside local laboratory normal limits, (b) the derangement was not present previously and (c) could not be explained by a concurrent medical condition. Abnormal laboratory results were investigated at clinician discretion. Laboratory results were evaluated based on their independent ability to detect relapse within 3 months of confirmation. Sensitivity, specificity, negative predictive value (NPV) and positive predictive value (PPV) were derived from $2 \times 2$ contingency tables and 95\% confidence intervals (Cls) were determined exactly.

In addition, receiver operating characteristics (ROC) and area under the curve (AUC) analysis were undertaken to determine the utility of ALC, AMC and LMR as a marker for relapse. AMC and ALC were evaluated as continuous variables, and LMR was calculated by dividing the ALC by the AMC. Survival analysis was performed using the Kaplan-Meier method and compared by the log-rank test between different groups. All values were two-sided and statistical significance was accepted at $p<0.05$. The study was approved by the local institutional review boards (LR117/2015).

\section{RESULTS}

Between January 2000 and January 2015, 346 eligible patients underwent 3048 outpatient visits. The median follow-up from CR1 was 30 months (range 3-184). Baseline demographics are detailed in Table 1. Laboratory investigations were performed at 2746 visits (90\%), with FBE being the most common test ordered (Table 2). $\mathrm{LDH}$ was predominantly performed in non-Hodgkin's lymphoma (NHL) and ESR in HL.

Relapse of lymphoma occurred in 56/346 (16\%) patients (33 DLBCL, $19 \mathrm{HL}, 4$ other). The median age at relapse was 64.3 years (range 18-91), and 51\% were over 60 years of age. Forty-three out of $56(77 \%)$ had advanced stage disease and 18/56 (32\%) were at high risk (as defined in Table 1). Only one patient (high-risk HL) received an abbreviated chemotherapy course; the remaining 45 patients received a full course of standard treatment. The median duration from treatment completion until relapse was 14 months (range 3-84 months), with $48 \%$ of relapses occurring in the first year, $31 \%$ in the second year and the remainder $(21 \%)$ occurring up to 7 years after the end of treatment.

\begin{tabular}{|c|c|c|c|c|c|c|}
\hline & \multicolumn{2}{|c|}{$\begin{array}{l}\text { All visits }(n= \\
3048)\end{array}$} & \multicolumn{2}{|c|}{$\mathrm{NHL}(n=1908)$} & \multicolumn{2}{|c|}{$\mathrm{HL}(n=1140)$} \\
\hline & Total & Abnormal & Total & Abnormal & Total & Abnormal \\
\hline Any labs & 2746 & 404 (15\%) & 1707 & 279 (16\%) & 1039 & 125 (12\%) \\
\hline FBE & 2660 & $271(10 \%)$ & 1638 & 195 (12\%) & 1022 & $76(7 \%)$ \\
\hline LDH & 2147 & $187(9 \%)$ & 1362 & $135(10 \%)$ & 785 & $52(7 \%)$ \\
\hline ESR & 411 & $25(6 \%)$ & - & - & 406 & $25(6 \%)$ \\
\hline
\end{tabular}


Table 3. Abnormal laboratory results in asymptomatic patients at scheduled appointments

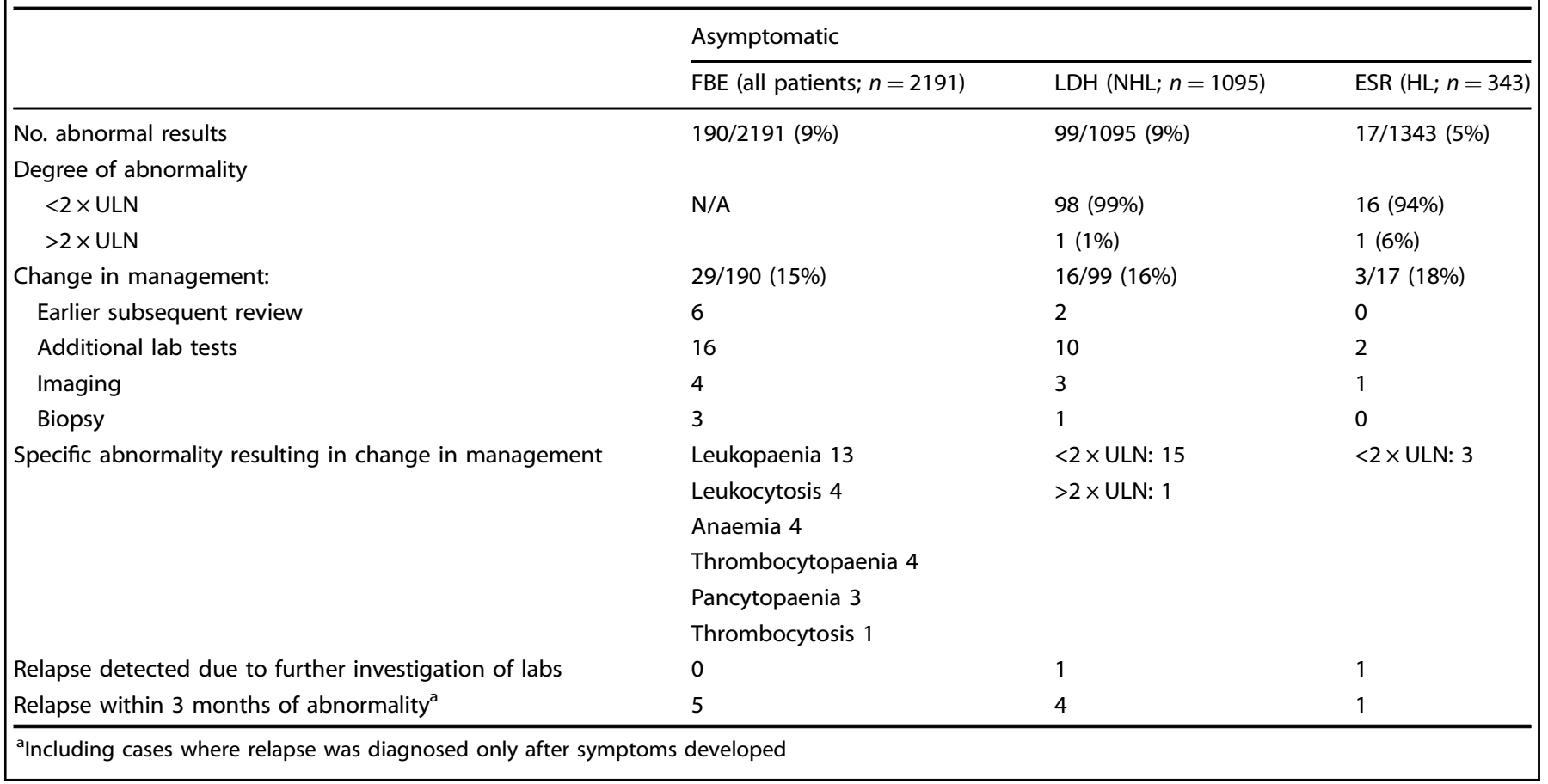

Table 4. Performance of testing

\begin{tabular}{llllllll}
\hline & All tests & & FBE & & LDH (NHL) & & ESR (HL) \\
\hline Sensitivity & 0.42 & $(31-52)^{\mathrm{a}}$ & 0.46 & $(35-57)$ & 0.28 & $(15-44)$ & 0.39 \\
Specificity & 0.87 & $(86-89)$ & 0.75 & $(73-76)$ & 0.89 & $(87-90)$ & 0.86 \\
PPV & 0.09 & $(7-13)$ & 0.05 & $(4-7)$ & 0.08 & $(4-13)$ & $(17-64)$ \\
NPV & 0.98 & $(87-93)$ & 0.98 & $(97-98)$ & 0.97 & $(96-98)$ & 0.11 \\
\hline
\end{tabular}

PPV positive predictive value, NPV negative predictive value. ${ }^{\text {a }} 95 \%$ confidence interval

Relapse was diagnosed by routine laboratory investigations in $3 / 56$ (5\%) and routine imaging in 10/56 (18\%) patients. Clinical symptoms/ signs lead to diagnosis of relapse in $43 / 56$ (80\%; 40 with symptoms, 3 with signs only); 19 of which were detected at unscheduled visits. Unscheduled appointments due to patient-reported symptoms ( $3 \%$ of all visits) showed a significantly stronger association with relapse than scheduled visits (odds ratio 50.4, $p<0.001$ ).

Abnormal laboratory results were recorded at 404/3048 followup visits: 304 in asymptomatic and 100 in symptomatic patients.

\section{Asymptomatic patients}

An unexplained abnormal result prompted a change in management at 46/304 (15\%) visits in asymptomatic patients: 19/46 (41\%) had repeat interval laboratory investigations only, 13/46 (28\%) underwent additional imaging, 10/46 (22\%) were booked for an earlier future review with repeat labs and 4/46 (9\%) had biopsies in addition to imaging. The specific laboratory abnormalities and associated changes in management in asymptomatic patients at scheduled appointments are described in Table 3. Almost all elevations in LDH and ESR were $<2$ times the upper limit of normal (ULN), and leukopaenia was the most common FBE abnormality $(12 / 29 ; 41 \%)$ resulting in change in management.

Relapse was diagnosed by $3 / 304$ (1\%) abnormal results in asymptomatic patients; one TCL with neutropaenia and thrombocytopaenia, and two HL patients; one with elevated LDH and one with elevated ESR. No relapses in NHL were diagnosed on the basis of an abnormal LDH alone.

In five additional patients, relapse was detected within 3 months of an abnormal result; however, in these cases, suspicion of relapse arose only after the patient developed symptoms. The abnormalities were: lymphopaenia, elevated LDH and both elevated LDH and abnormal FBE in three patients.

Symptomatic patients

In contrast, $67 / 100(67 \%)$ of symptomatic patients with an abnormal result underwent a change in their management. The most common changes were further imaging $(n=35 ; 52 \%)$ and imaging with biopsy $(n=17 ; 25 \%)$, followed by earlier future review and labs $(n=7 ; 10 \%)$ and repeat interval laboratory investigations only ( $n=8 ; 12 \%)$.

The sensitivity, specificity, PPV and NPV of all routine lab tests (FBE, LDH and ESR combined) in detecting relapse was $42 \%, 87 \%$, $9 \%$ and $98 \%$, respectively. Performance characteristics of individual lab tests are detailed in Table 4. The PPV of LDH remained the same even in the subset of $115 \mathrm{NHL}$ patients with elevated baseline $\mathrm{LDH}(8 \%$, confidence interval $(\mathrm{Cl}), 5-12)$. In the $43 \mathrm{HL}$ patients with an elevated baseline ESR, the PPV of ESR was even lower $(6.5 \%, \mathrm{Cl}, 2-17)$.

ROC and AUC analysis showed that ALC, AMC and LMR at each appointment $(n=2660)$ were all very poor markers for relapse 


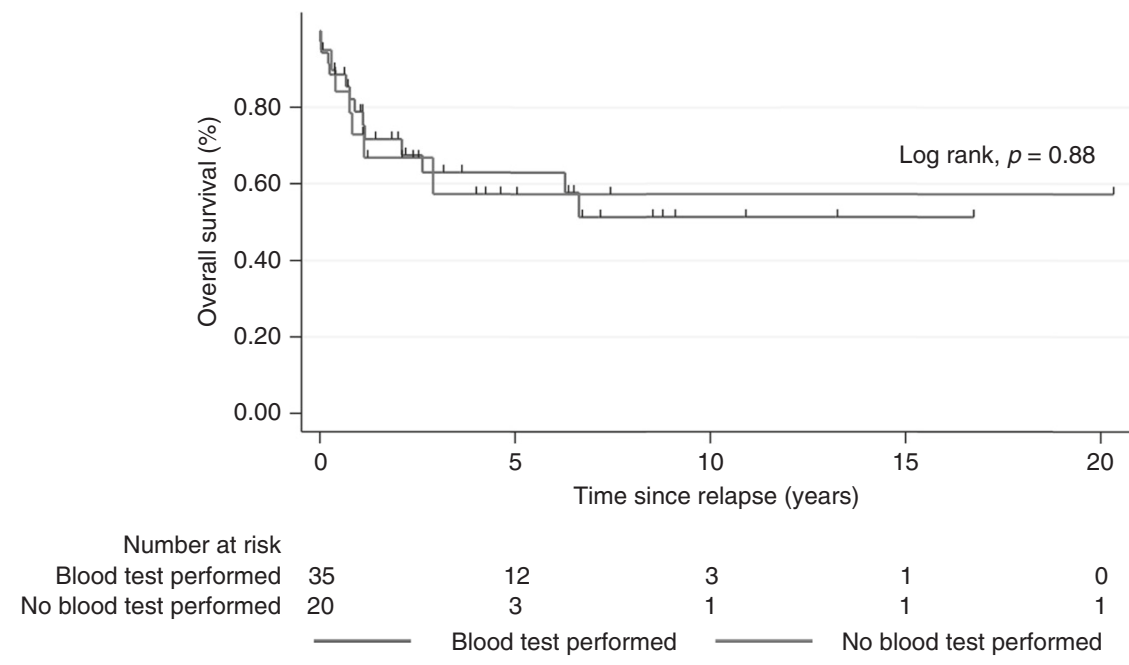

Fig. 1 Survival curve in relapsed patients. This shows the post-relapse survival based on whether a blood test was performed in preclinical period (3 months) for relapse

(AUC $=0.517,0.529$ and 0.577 respectively); thus, their performance characteristics were not calculated.

Two-year OS and EFS were $76 \%(95 \% \mathrm{Cl}, 71-80)$ and $70 \%(95 \%$ $\mathrm{Cl}, 65-80)$, respectively, in the whole cohort. There was no significant difference in post-relapse survival between patients who had laboratory investigations performed $\leq 3$ months prior to documented relapse versus patients who did not ( $p=0.88$, Fig. 1$)$.

\section{DISCUSSION}

This analysis, from one of the largest and most comprehensive series in the modern era to our knowledge, demonstrates little benefit of including routine laboratory testing to detect relapse in follow-up of asymptomatic patients with aggressive lymphoma achieving metabolic CR after first-line chemotherapy. In line with published reports, our results confirm that clinical symptoms and signs are the single most important predictor of relapse, ${ }^{16,17}$ with $80 \%$ of relapsed patients having symptoms at presentation and only $1 \%$ of isolated abnormal blood test results leading to a diagnosis of relapse. There was no difference in survival between patients who had blood tests and those who did not.

Previous studies have reported that routine blood tests do not reliably predict relapse. ${ }^{17-19}$ However, all have assessed either only one parameter or 'blood tests' as a whole without describing which tests were performed or omitted. Our study is the only one to assess the performance of individual tests, their role in the detection of relapse and their impact on management and overall outcomes in a population with PET-confirmed CR at 3 months.

ESR had been proposed as a useful marker of relapse in $\mathrm{HL}$ in $1991^{20}$, but subsequent studies dispute this, with the vast majority of relapses detected by clinical findings rather than by ESR alone. $^{21,22}$ Nevertheless, ESR is still frequently performed during follow-up. In our cohort, ESR had a sensitivity of only $39 \%$ for detection of relapse in $\mathrm{HL}$, and only one relapse was diagnosed by an isolated elevated ESR.

$\mathrm{LDH}$ has also been proposed as a useful screening test for relapse in DLBCL in the pre-rituximab era ${ }^{23}$, but recent studies are consistent with ours in showing its lack of predictive value in the absence of symptoms or signs suggesting relapse. ${ }^{13,18,24,25}$ The PPV of an elevated LDH in our aggressive NHL cohort was $8 \%$, even after accounting for known causes of LDH elevation such as liver disease and infection. No relapses in $\mathrm{NHL}$ were detected on the basis of LDH alone. Our findings confirm results from a previous smaller series of 100 DLBCL patients, ${ }^{19}$ which analysed $\mathrm{LDH}$ at every appointment and reported a low PPV of $9 \%$ and sensitivity of $47 \%$ for relapse. Interestingly, LDH was ordered at $69 \%$ of HL follow-up appointments, despite a lack of evidence or recommendations by guidelines for its use in monitoring this subtype, and lead to the detection of one $\mathrm{HL}$ relapse.

FBE was the most commonly performed test in our study, with an abnormal result in $10 \%$ of samples, yet was associated with a change in management in $<15 \%$ of the time. There was one relapse diagnosed on the basis of FBE alone. These findings are also consistent with the literature, with several studies reporting no relapses detected by FBE abnormalities. ${ }^{21-23}$

Baseline lymphocyte and monocyte counts and the LMR have prognostic value for both $\mathrm{DLBCL}^{26}$ and $\mathrm{HL}^{27}$ and three retrospective studies concluded that a low ALC and LMR during followup is a useful indicator of relapse in DLBCL. PPV and NPV in these studies ranged between $68-74 \%$ and $49-96 \%$, respectively, with sensitivity $68-89 \%$ and specificity $88 \% .^{11-13}$ However, these studies analysed parameters at a single time point just prior to relapse without accounting for symptomatology or confirming initial CR on PET. In contrast, our analysis demonstrated that ALC, AMC and LMR had almost no ability to discriminate between relapsed and non-relapsed patients, with far lower AUC values than previously reported ( 0.52 versus 0.91 for $A L C){ }^{13}$

It may be argued that the NPV of laboratory tests was high in our study (98\%) and provides reassurance to patients with normal results. Conversely, $15 \%$ of blood tests had an unexplained abnormality; not only are they of poor PPV in asymptomatic patients, they almost always result in unnecessary patient anxiety and often lead to further investigations, which are seldom abnormal. Routine blood tests have been postulated as a method of monitoring for therapy-related myelodysplastic syndromes (MDS). The incidence of therapy-related MDS in patients receiving induction chemotherapy for aggressive lymphomas is only marginally higher than the general population $(0.4-1.2 \%$ post treatment versus $0.3 \%$ in the general population ${ }^{28-30}$ ). More importantly, there is limited evidence for early detection of MDS in asymptomatic patients and current guidelines do not recommend treatment for the majority of this cohort. Additionally, screening for MDS would, at most, warrant a FBE alone, but not other currently recommended blood tests in lymphoma surveillance guidelines.

Recognising this study is retrospective, and the design remains robust. The patients were treated uniformly, as demonstrated by the high percentage of patients undergoing the individual blood tests, consistent use of end of treatment PET to confirm metabolic remission and limited variation in treatment regimens. Unlike the majority of prior analyses, ${ }^{17-19}$ this study reviewed all labs 
performed for the duration of follow-up in patients with PETconfirmed CR for at least 3 months following treatment. Our study included all major histological subtypes of aggressive lymphoma and is likely relevant to a wider population. Although of note, the exclusion of primary refractory disease in our cohort to accurately analyse the role of blood tests in detection of relapse led to a lower proportion of high-risk patients than many published series.

This study confirms that common blood tests do not reliably detect relapse of aggressive lymphoma in asymptomatic patients treated in the modern era and should not be recommended by current international guidelines. They are no longer performed in this context in our institutions. More novel methods of relapse detection such as circulating tumour DNA have demonstrated greater specificity and sensitivity than standard blood parameters; however, this technology is yet to be widely available and affordable.

\section{AUTHOR CONTRIBUTIONS}

E.A.H. and A.G. designed the study, Z.L. and O.E. performed the research and F.J.H. and M.G. contributed additional data. Z.L. analysed the data, Z.L. and E.A.H. wrote the paper and A.G. and G.C. critically revised the paper. All authors approved the final version.

\section{ADDITIONAL INFORMATION}

Ethics approval and consent to participate: This study was approved by the local institutional review boards. Austin Health Human Research Ethics Committee, reference number LNR/15/AUSTIN/432. Eastern Health Human Research Ethics Committee, reference number LR117/2015

Availability of data and material: Materials, data and associated protocols are available from the corresponding author on reasonable request.

Competing interests: The authors declare no competing interests.

Note: This work is published under the standard license to publish agreement. After 12 months the work will become freely available and the license terms will switch to a Creative Commons Attribution 4.0 International (CC BY 4.0).

Publisher's note:Springer Nature remains neutral with regard to jurisdictional claims in published maps and institutional affiliations.

\section{REFERENCES}

1. Cunningham, D. et al. Rituximab plus cyclophosphamide, doxorubicin, vincristine, and prednisolone in patients with newly diagnosed diffuse large B cell nonHodgkin lymphoma: a phase 3 comparison of dose intensification with 14-day versus 21-day cycles. Lancet 381, 1817-1826 (2013).

2. Johnson, P., Trotman, J. \& Federico, M. Interim PET-CT scan in advanced Hodgkin's lymphoma. N. Engl. J. Med. 375, 999-100 (2016).

3. Gleeson, M., Peckitt, C., To, Y. M., \& Edwards, L. CHOP versus GEM-P in the first-line treatment of t-cell lymphoma (ptcl): initial results of the UK NCRI phase II randomised chemo-t trial. Haematol. Oncol. 35(Suppl. 2), 75-76 (2017).

4. National Comprehensive Cancer Network: The NCCN Non-Hodgkin's Lymphomas Clinical Practice Guidelines in Oncology (Version 2.2015): http://www.nccn.org

5. El-Galaly, T. C. et al. Role of routine imaging in detecting recurrent lymphoma: A review of 258 patients with relapsed aggressive non-Hodgkin and Hodgkin lymphoma. Am. J. Hematol. 89, 575-580 (2014).

6. Thompson, C. A. et al. Utility of routine post-therapy surveillance imaging in diffuse large B-cell lymphoma. J. Clin. Oncol. 32, 3506-3512 (2014).

7. Eichenauer, D. A. et al. Hodgkin's lymphoma: ESMO Clinical Practice Guidelines for diagnosis, treatment and follow-up. Ann. Oncol. 25(Suppl. 3), iii70-iii75 (2014).
8. Zelenetz, A. D. \& Hoppe, R. T. Panel NN-HsLPG. NCCN: Non-Hodgkin's lymphoma. Cancer Control 8(Suppl. 2), 102-113 (2001).

9. Tilly, H. et al. Diffuse large B-cell lymphoma (DLBCL): ESMO Clinical Practice Guidelines for diagnosis, treatment and follow-up. Ann. Oncol. 26(Suppl. 5), v116-v125 (2015).

10. National Comprehensive Cancer Network: The NCCN Hodgkin Lymphoma Clinical Practice Guidelines in Oncology (Version 2.2015): http://www.nccn.org

11. Wei, X. et al. Lymphopenia predicts preclinical relapse in the routine follow-up of patients with diffuse large B-cell lymphoma. Leuk. Lymphoma 56, 1261-1265 (2015).

12. Yan-Li, L., Kang-Sheng, G., Yue-Yin, P., Yang, J. \& Zhi-Min, Z. The lower peripheral blood lymphocyte/monocyte ratio assessed during routine follow-up after standard first-line chemotherapy is a risk factor for predicting relapse in patients with diffuse large B-cell lymphoma. Leuk. Res. 38, 323-328 (2014).

13. Porrata, L. F. et al. Lymphopenia assessed during routine follow-up after immunochemotherapy (R-CHOP) is a risk factor for predicting relapse in patients with diffuse large B-cell lymphoma. Leukemia 24, 1343-1349 (2010).

14. Bauml, J. M. et al. Scan-associated distress in lung cancer: quantifying the impact of "scanxiety". Lung Cancer 100, 110-113 (2016).

15. Thompson, C. A. et al. Surveillance CT scans are a source of anxiety and fear of recurrence in long-term lymphoma survivors. Ann. Oncol. 21, 2262-2266 (2010).

16. Bestawros, A., Foltz, L., Srour, N., Savage, K. J. \& Connors, J. M. Patients' and physicians' roles in detecting recurrent Hodgkin lymphoma following complete remission. Ann. Oncol. 24, 1359-1363 (2013).

17. Chasty B. et al. Lack of effectiveness of routine clinic and blood test-based followup for diffuse large B cell lymphoma. Br. J. Haematol. https://doi.org/10.1111/ bjh.14745 (2017).

18. El-Sharkawi, D. et al. Elevated lactate dehydrogenase levels detected during routine follow-up do not predict relapse in patients with diffuse large B-cell lymphoma who achieve complete remission after primary treatment with rituximab, cyclophosphamide, doxorubicin, vincristine and prednisone-like immunochemotherapy. Leuk. Lymphoma 53, 1949-1952 (2012).

19. Hong, J. et al. Prognostic role of serum lactate dehydrogenase beyond initial diagnosis: a retrospective analysis of patients with diffuse large B cell lymphoma. Acta Haematol. 130, 305-311 (2013).

20. Henry-Amar, M. et al. Erythrocyte sedimentation rate predicts early relapse and survival in early-stage Hodgkin disease. The EORTC Lymphoma Cooperative Group. Ann. Intern. Med. 114, 361-365 (1991).

21. Torrey, M. J., Poen, J. C. \& Hoppe, R. T. Detection of relapse in early-stage Hodgkin's disease: role of routine follow-up studies. J. Clin. Oncol. 15, 1123-1130 (1997).

22. Radford, J. A., Eardley, A., Woodman, C. \& Crowther, D. Follow up policy after treatment for Hodgkin's disease: too many clinic visits and routine tests? A review of hospital records. BMJ 314, 343-346 (1997).

23. Weeks, J. C., Yeap, B. Y., Canellos, G. P. \& Shipp, M. A. Value of follow-up procedures in patients with large-cell lymphoma who achieve a complete remission. J. Clin. Oncol. 9, 1196-1203 (1991).

24. Hiniker, S. M. et al. Value of surveillance studies for patients with stage I to II diffuse large B-cell lymphoma in the rituximab era. Int. J. Radiat. Oncol. Biol. Phys. 92, 99-106 (2015).

25. William, B. M. et al. The utility of lactate dehydrogenase in the follow up of patients with diffuse large B-cell lymphoma. Rev. Bras. Hematol. Hemoter. 35, 189-191 (2013).

26. Wilcox, R. A. et al. The absolute monocyte and lymphocyte prognostic score predicts survival and identifies high-risk patients in diffuse large-B-cell lymphoma. Leukemia 25, 1502-1509 (2011).

27. Porrata, L. F. et al. Peripheral blood lymphocyte/monocyte ratio at diagnosis and survival in classical Hodgkin's lymphoma. Haematologica 97, 262-269 (2012).

28. Moser, E. C. et al. Risk of second cancer after treatment of aggressive nonHodgkin's lymphoma; an EORTC cohort study. Haematologica 91, 1481-1488 (2006).

29. Sacchi, S. et al. Second malignancies after treatment of diffuse large B-cell nonHodgkin's lymphoma: a GISL cohort study. Haematologica 93, 1335-1342 (2008).

30. Bari, A. et al. Therapy-related myeloid neoplasm in non-Hodgkin lymphoma survivors. Mediterr. J. Hematol. Infect. Dis. 3, e2011065 (2011). 